\title{
Multi-detector CT imaging: impact of virtual tube current reduction and sparse sampling on detection of vertebral fractures
}

\author{
Nico Sollmann ${ }^{1,2}$ (D) Kai Mei ${ }^{3} \cdot$ Dennis M. Hedderich ${ }^{1} \cdot$ Christian Maegerlein $^{1} \cdot$ Felix K. Kopp ${ }^{3} \cdot$ Maximilian T. Löffler $^{1}$ • \\ Claus Zimmer ${ }^{1}$. Ernst J. Rummeny ${ }^{3} \cdot$ Jan S. Kirschke ${ }^{1} \cdot$ Thomas Baum $^{1} \cdot$ Peter B. Noël ${ }^{3,4}$
}

Received: 18 October 2018 / Revised: 30 January 2019 / Accepted: 8 February 2019 / Published online: 22 March 2019

(C) The Author(s) 2019

\begin{abstract}
Purpose To systematically evaluate the effects of virtual tube current reduction and sparse sampling on image quality and vertebral fracture diagnostics in multi-detector computed tomography (MDCT).

Materials and methods In routine MDCT scans of 35 patients $(80.0 \%$ females, $70.6 \pm 14.2$ years, $65.7 \%$ showing vertebral fractures), reduced radiation doses were retrospectively simulated by virtually lowering tube currents and applying sparse sampling, considering $50 \%, 25 \%$, and $10 \%$ of the original tube current and projections, respectively. Two readers evaluated items of image quality and presence of vertebral fractures. Readout between the evaluations in the original images and those with virtually lowered tube currents or sparse sampling were compared.

Results A significant difference was revealed between the evaluations of image quality between MDCT with virtually lowered tube current and sparse-sampled MDCT $(p<0.001)$. Sparse-sampled data with only $25 \%$ of original projections still showed good to very good overall image quality and contrast of vertebrae as well as minimal artifacts. There were no missed fractures in sparse-sampled MDCT with 50\% reduction of projections, and clinically acceptable determination of fracture age was possible in MDCT with 75\% reduction of projections, in contrast to MDCT with $50 \%$ or $75 \%$ virtual tube current reduction, respectively.

Conclusion Sparse-sampled MDCT provides adequate image quality and diagnostic accuracy for vertebral fracture detection with $50 \%$ of original projections in contrast to corresponding MDCT with lowered tube current. Thus, sparse sampling is a promising technique for dose reductions in MDCT that could be introduced in future generations of scanners.

Key Points

- MDCT with a reduction of projection numbers of 50\% still showed high diagnostic accuracy without any missed vertebral fractures.

- Clinically acceptable determination of vertebral fracture age was possible in MDCT with a reduction of projection numbers of $75 \%$.

- With sparse sampling, higher reductions in radiation exposure can be achieved without compromised image or diagnostic quality in routine MDCT of the spine as compared to MDCT with reduced tube currents.
\end{abstract}

Keywords Bone fractures $\cdot$ Multi-detector computed tomography $\cdot$ Osteoporosis $\cdot$ Radiation exposure $\cdot$ Spine

Electronic supplementary material The online version of this article (https://doi.org/10.1007/s00330-019-06090-2) contains supplementary material, which is available to authorized users.

\section{Nico Sollmann}

Nico.Sollmann@tum.de

1 Department of Diagnostic and Interventional Neuroradiology, Klinikum rechts der Isar, Technische Universität München, Ismaninger Str. 22, 81675 Munich, Germany

2 TUM-Neuroimaging Center, Klinikum rechts der Isar, Technische Universität München, Munich, Germany
3 Department of Diagnostic and Interventional Radiology, Klinikum rechts der Isar, Technische Universität München, Ismaninger Str. 22, 81675 Munich, Germany

4 Department of Radiology, Perelman School of Medicine, University of Pennsylvania, 3400 Spruce Street, One Silverstein, Philadelphia, PA 19104, USA 


$\begin{array}{ll}\text { Abbreviations } \\ \text { CT } & \text { Computed tomography } \\ \text { FBP } & \text { Filtered back projection } \\ \text { FOV } & \text { Field of view } \\ \text { ICC } & \text { Intraclass correlation coefficient } \\ \text { IR } & \text { Iterative reconstruction } \\ \text { MDCT } & \text { Multi-detector computed tomography } \\ \text { MRI } & \text { Magnetic resonance imaging } \\ \text { PACS } & \text { Picture Archiving and Communication System } \\ \text { R1 } & \text { Reader 1 } \\ \text { R2 } & \text { Reader 2 }\end{array}$

\section{Introduction}

Vertebral fractures are frequent in clinical routine and are primarily observed in the context of injuries or as major manifestations of osteoporosis, even in the absence of any obvious trauma [1-3]. Spine radiography is commonly applied for the detection of suspected vertebral fractures; however, it has been shown that computed tomography (CT) is superior by reducing the risk of missing a fracture, thus resulting in a higher sensitivity and specificity with fracture detection rates of 97 to $100 \%$ at the spine [4-6].

The increased use of CT instead of radiography for the purpose of improved diagnostics comes at the cost of elevated radiation exposure for the patient: one-time scanning with a modern CT scanner applies an estimated effective dose of $5.6 \mathrm{mSv}$ and $10.0 \mathrm{mSv}$ for the lumbar and whole dorsal spine, respectively $[7,8]$. The use of $\mathrm{CT}$ entails an estimated cancer risk ratio that is multifold higher than in radiography, and it can further increase due to cumulative effects when additional imaging is performed [7-9]. Thus, CT with reduced radiation exposure, but without simultaneous constraints for image quality or diagnostic accuracy seems crucial.

Despite its clinical relevance, previous research on dose reductions in $\mathrm{CT}$ at the spine is generally scarce. In vivo, radiation exposure reductions have been achieved by lowered tube current or voltage at the level of the cervical spine, resulting in largely preserved image quality except for the lower cervical spine [10,11]. Recently, iterative reconstruction (IR) algorithms have been applied together with low-dose $\mathrm{CT}$, but led to worse image quality for soft tissue and cervical vertebrae when compared to standard-dose $\mathrm{CT}$ using filtered back projection (FBP) [12]. To date, only few studies investigated CT with reduced doses specifically for diagnostics of vertebral fractures, showing that low-dose CT with IR may maintain a high diagnostic performance compared to standard-dose CT with IR in trauma patients [13, 14].

In addition to lowering tube current or voltage to reduce $\mathrm{x}$ ray exposure during $\mathrm{CT}$, the number of acquired projections can be decreased with sparse-sampled acquisition schemes. Reducing projection views is a promising strategy since lowering the number of projections can clearly reduce the radiation dose, with previous research indicating a high potential of this approach resulting in reasonable image quality [15-20]. However, sparse sampling has not been applied at the spine for fracture diagnostics yet.

Against this background, the aim of this study is to evaluate the effects of virtual tube current reduction and sparse sampling on image quality and vertebral fracture diagnostics in multi-detector CT (MDCT). Our hypothesis is that MDCT with sparse sampling would provide better image and diagnostic quality when compared to MDCT with virtual lowering of tube current and, thus, might allow for more drastic reductions in radiation exposure.

\section{Materials and methods}

\section{Patients}

This retrospective study was approved by the local institutional review board (registration number: 62/18S) and was conducted in accordance with the Declaration of Helsinki. Overall, 35 patients were included $(80.0 \%$ females, mean age: $70.6 \pm 14.2$ years, age range: $26.2-93.1$ years), with 23 patients $(65.7 \%)$ showing at least one vertebral fracture (fracture group) and 12 patients (34.3\%) showing no vertebral fracture (control group). Eligible patient cases were identified in the institutional digital Picture Archiving and Communication System (PACS) between November 2016 and November 2017.

Inclusion criteria were (1) availability of routine MDCT of the spine (irrespective of the initial suspected diagnosis or distinct clinical symptoms leading to MDCT), (2) additional spinal magnetic resonance imaging (MRI) performed prior or subsequent to MDCT (including short-tau inversion recovery sequences; only for the fracture group), and (3) diagnosis of at least one vertebral fracture according to MDCT (only for the fracture group). Exclusion criteria for both the fracture and control group were (1) age below 18 years, (2) movement artifacts in imaging data, (3) malignant bone lesions (e.g., bone metastases), (4) any history of metabolic bone disorders aside from osteoporosis, and (5) any implants captured by the field of view (FOV).

\section{Multi-detector computed tomography}

All scans were performed with a 64-channel MDCT scanner (Brilliance 64; Philips Healthcare). Parameters of the scanning protocol are shown in Table 1. X-ray tube current was modulated implicitly by the scanner during the helical scan according to the examined body part and estimated body size as derived from the scout scan. All examinations were performed without administration of a contrast agent. 
Table 1 Scan parameters and image reconstruction

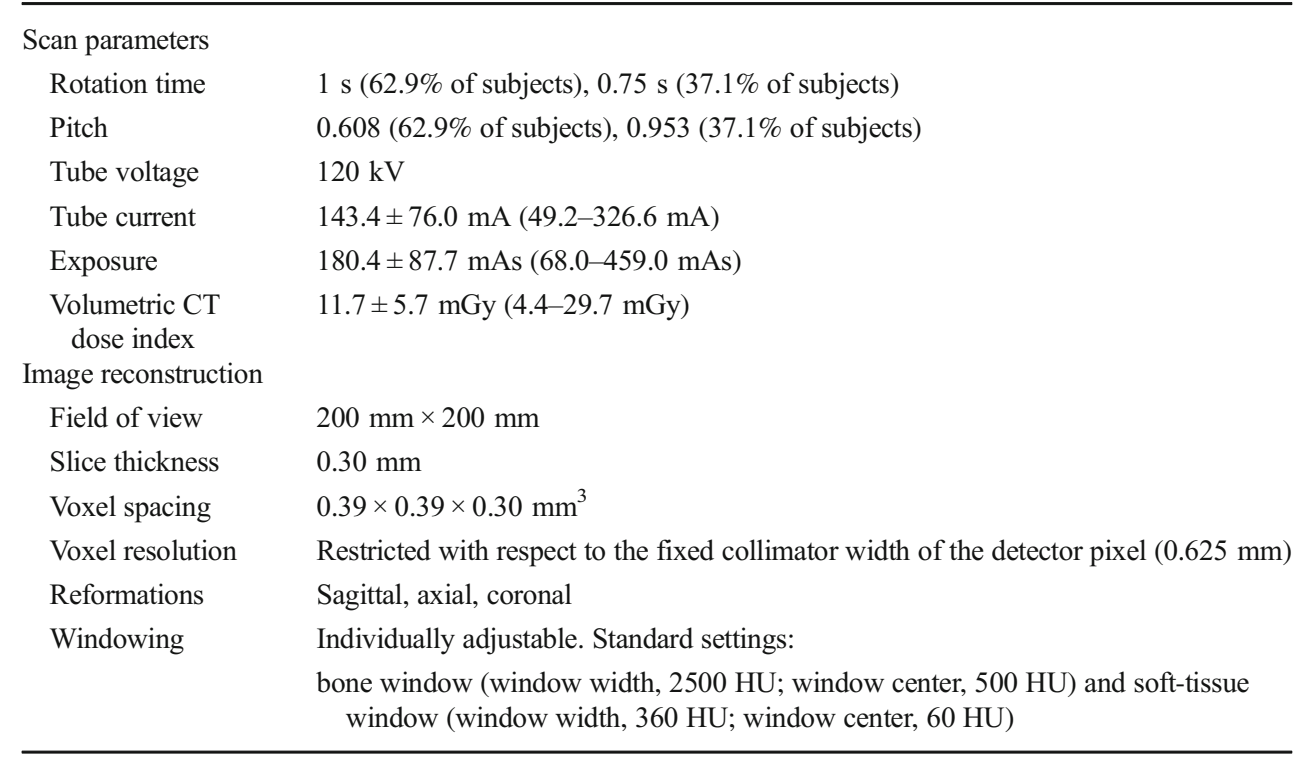

\section{Virtual tube current reduction and sparse sampling}

Based on raw projection data, we used a simulation algorithm to generate lower tube currents for MDCT scans [16, 21-23]. System parameters of the scanner were considered and electronic noise was calibrated for each pixel at the detector. Simulations were generated as if the scans were made at $50 \%$ (D50 P100), 25\% (D25 P100), and 10\% (D10 P100) of the original $\mathrm{X}$-ray tube current and used for image evaluation, in addition to the original imaging data defined as D100 P100. Furthermore, sparse sampling was applied at levels of $50 \%$ (D100 P50), 25\% (D100 P25), and 10\% (D100 P10) of the original projection data, which was achieved by reading every second, fourth, and tenth projection angle and deleting the remaining projections in the sinogram [16, 23, 24]. While the projection number per full rotation was lowered, other parameters, including patient location and projection geometry, were not changed. All images were reconstructed using FBP and a standard Ram-Lak filter $[25,26]$. Table 1 provides an overview of image reconstruction parameters.

\section{Image evaluation}

Two board-certified radiologists ( 6 and 8 years of experience in radiology) evaluated all imaging data (35 patients $\times 7$ imaging datasets per patient $=245$ datasets for evaluation for reader 1 [R1] and reader 2 [R2], respectively), which were uploaded and stored in IntelliSpace Portal (version 9.0; Philips Healthcare) for visualization and evaluation.

First, both readers evaluated the original images with $100 \%$ tube current and projections (D100 P100) as the clinical standard in consensus with the MRI available to confirm the diagnosis of an acute or old vertebral fracture. Then, both readers independently evaluated the remaining datasets in random order (D50 P100, D25 P100, D10 P100, D100 P50, $\mathrm{D} 100 \mathrm{P} 25$, and D100 P10), assessing images derived from the same tube current or number of projections within 1 day for all patients and sticking to an interval of at least 7 days before continuing with images of another tube current or number of projections. The order of patient cases was also randomized for each tube current and number of projections, with the readers being blinded to all clinical patient data, the evaluations of each other, the assignments of patients to the fracture or control group, and all previous evaluations performed.

During evaluations, the number of vertebral fractures per patient had to be determined first, with the vertebrae included in the FOV being provided for each case to allow assignment of single fractures to specific vertebrae. Then, the items and scores presented in Table 2 were considered.

\section{Statistical analyses}

For statistical analyses and generation of graphs, SPSS (version 20.0; IBM SPSS Statistics for Windows, IBM Corp.) was used. A $p$ value $<0.05$ was considered statistically significant.

Descriptive statistics were calculated for patient demographics and all items of evaluation. Wilcoxon signed-rank tests were performed to compare overall image quality, overall artifacts, contrast of vertebrae, and diagnostic confidence between MDCT with virtually lowered tube current and sparsesampled images, i.e., D50 P100 vs. D100 P50, D25 P100 vs. D100 P25, and D10 P100 vs. D100 P10, respectively. Furthermore, overall image quality, overall artifacts, and contrast of vertebrae of D100 P100 as the gold standard were compared with MDCT with virtually lowered tube current and sparse-sampled images using Wilcoxon signed-rank tests, which were achieved separately for each reader. 
Table 2 Scoring system for the evaluation of image quality and vertebral fractures

\begin{tabular}{|c|c|c|c|c|c|}
\hline \multicolumn{6}{|c|}{ Overall image evaluation } \\
\hline \multirow[t]{2}{*}{ Item } & Score & & & & \multirow[b]{2}{*}{5} \\
\hline & 1 & 2 & 3 & 4 & \\
\hline $\begin{array}{l}\text { Overall image } \\
\text { quality }\end{array}$ & $\begin{array}{l}\text { Very good to perfect quality } \\
\text { No compromise of } \\
\text { diagnostic quality }\end{array}$ & $\begin{array}{l}\text { Good to very good quality } \\
\text { No compromise of } \\
\text { diagnostic quality }\end{array}$ & $\begin{array}{l}\text { Medium quality } \\
\text { Acceptable diagnostic } \\
\text { quality }\end{array}$ & $\begin{array}{l}\text { Poor quality } \\
\text { Unacceptable } \\
\text { diagnostic quality }\end{array}$ & $\begin{array}{l}\text { Vertebrae not } \\
\text { distinguishable }\end{array}$ \\
\hline Overall artifacts & $\begin{array}{l}\text { No artifacts } \\
\text { No compromise of } \\
\text { diagnostic quality }\end{array}$ & $\begin{array}{l}\text { Minimal artifacts } \\
\text { No compromise of } \\
\text { diagnostic quality }\end{array}$ & $\begin{array}{l}\text { Prominent artifacts } \\
\text { Acceptable diagnostic } \\
\text { quality }\end{array}$ & $\begin{array}{l}\text { Major artifacts } \\
\text { Unacceptable } \\
\text { diagnostic quality }\end{array}$ & $\begin{array}{l}\text { Vertebrae not } \\
\text { distinguishable }\end{array}$ \\
\hline \multirow[t]{2}{*}{$\begin{array}{r}\text { Contrast of } \\
\text { vertebrae }\end{array}$} & $\begin{array}{l}\text { Very good to perfect } \\
\text { contrast }\end{array}$ & Good to very good contrast & Medium contrast & Poor contrast & \multirow[t]{2}{*}{$\begin{array}{l}\text { Vertebrae not } \\
\text { distinguishable }\end{array}$} \\
\hline & $\begin{array}{l}\text { No compromise of } \\
\text { diagnostic quality }\end{array}$ & $\begin{array}{l}\text { No compromise of } \\
\text { diagnostic quality }\end{array}$ & $\begin{array}{l}\text { Acceptable diagnostic } \\
\text { quality }\end{array}$ & $\begin{array}{l}\text { Unacceptable } \\
\text { diagnostic quality }\end{array}$ & \\
\hline \multicolumn{6}{|c|}{ Fracture evaluation } \\
\hline \multirow[t]{2}{*}{ Item } & Score & & & & \\
\hline & 1 & 2 & & 3 & \\
\hline $\begin{array}{l}\text { Diagnostic } \\
\text { confidence }\end{array}$ & High confidence & Medium confidence & & Low confidence & \\
\hline Age of fracture & Acute & Unclear/not distinguishable & & Old & \\
\hline
\end{tabular}

Interreader intraclass correlation coefficients (ICCs) were calculated for overall image quality, overall artifacts, contrast of vertebrae, and diagnostic confidence in MDCT with virtually lowered tube current and sparse sampling, respectively $[27,28]$. As a measure of agreement between imaging with virtual lowering of tube current and sparse sampling, Cohen's kappa coefficients were determined for age of fracture. Moreover, weighted Cohen's kappa coefficients were determined between the results of both readers for age of fracture [29-31].

\section{Results}

Virtual lowering of tube current and sparse sampling were successfully achieved in all patients (Figs. 1 and 2). A median of eight vertebrae (range 4-19 vertebrae) was captured by the FOV of MDCT scans, which covered the cervical spine in $20.0 \%$, the cervico-thoracic spine in $8.6 \%$, the thoracic spine in $5.7 \%$, the thoraco-lumbar spine in $28.6 \%$, and the lumbar spine in $37.1 \%$. The average volumetric $\mathrm{CT}$ dose index recorded in the dose reports was $11.7 \pm 5.7 \mathrm{mGy}$ for original MDCT scans (Table 1), and was amounted $5.9 \mathrm{mGy}$, $2.9 \mathrm{mGy}$, and $1.2 \mathrm{mGy}$ for MDCT with virtually lowered tube current or sparse sampling at $50 \%, 25 \%$, and $10 \%$ of original data, respectively.

Both readers correctly identified all patients of the control group (34.3\% of included patients) without any assignments of vertebral fractures to controls in MDCT with virtually lowered tube current or sparse sampling. Among patients of the fracture group ( $65.7 \%$ of included patients), a total of 48 vertebral fractures was observed in the original MDCT with $100 \%$ tube current and projections (D100 P100). Patients of the fracture group showed a median of two vertebral fractures (range 1-6 vertebral fractures). These fractures affected the cervical spine in $10.0 \%$, the thoracic spine in $40.0 \%$, and the lumbar spine in $50.0 \%$. Based on original MDCT and MRI scanning, vertebral fractures were diagnosed as acute in $58.3 \%$ and old in $41.7 \%$.

\section{Overall image evaluation}

Both virtual tube current reduction and sparse sampling led to decreased overall image quality, increased overall artifacts, and reduced contrast of vertebrae according to the evaluation of both readers (Table 3, Figs. 1 and 2, Supplementary Fig. 1). The assessed parameters were significantly different in MDCT with virtually lowered tube current and sparsesampled datasets as compared to those in D100 P100 $(p<0.001$; D100 P100 vs. D50 P100/D25 P100/D10 P100 and D100 P100 vs. D100 P50/D100 P25/D100 P10 of both readers).

When comparing MDCT with virtually lowered tube current to sparse-sampled datasets for overall image quality, sparse sampling resulted in significantly better scores according to each reader for all comparisons $(p<0.001$, D50 P100 vs. D100 P50, D25 P100 vs. D100 P25, and D10 P100 vs. D100 P10 of both readers; Table 3 and Fig. 3). Similar findings with better scores for sparse-sampled imaging than for MDCT with virtually lowered tube current were observed for overall artifacts $(p<0.001$, except for D50 P100 vs. D100 P50 for R2: $p=0.20$; Table 3 and Fig. 3 ) and contrast of vertebrae $(p<0.001$, except for D25 P100 vs. D100 P25 for R2: $p=$ 0.005; Table 3 and Fig. 3). Good interreader agreement was observed for overall image quality, overall artifacts, and 


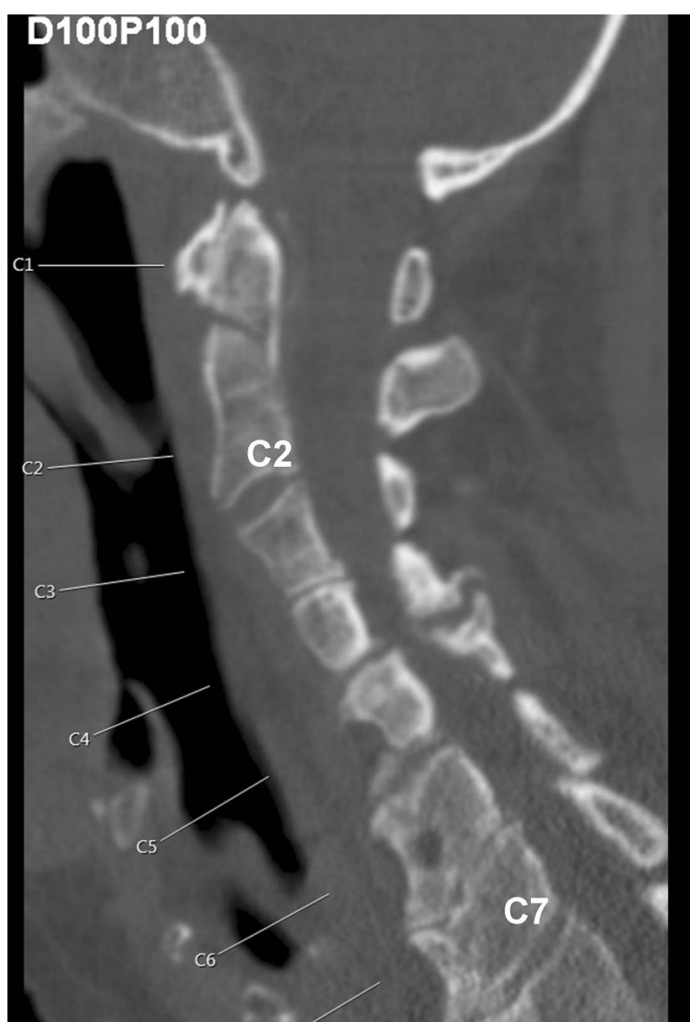

Fig. 1 Virtual tube current reduction and sparse sampling in multidetector CT (MDCT) of the cervical spine. Sagittal slices derived from full-dose MDCT (D100 P100), MDCT with virtually lowered tube current (D50 P100, D25 P100, and D10 P100), and MDCT with sparse sampling (D100 P50, D100 P25, and D100 P10) are shown in a patient with a cervical fracture $(\mathrm{C} 2$, dens fracture) contrast of vertebrae, respectively (ICC $>0.80$, R1 vs. R2 for D50 P100/D25 P100/D10 P100 and D100 P50/D100 P25/ D100 P10; Table 3).

\section{Fracture evaluation}

Virtual tube current reduction by $50 \%$ of original current (D50 P100) allowed for correct detection of 100\% (R1) and $95.8 \%$ (R2) of vertebral fractures when compared to original MDCT. Further lowering to $10 \%$ of original current (D10 P100) resulted in correct detection of 79.2\% (R1) and $87.5 \%$ (R2) of vertebral fractures (Table 4). Sparse-sampled MDCT with $50 \%$ of the original projections (D100 P50) allowed for correct detection of all vertebral fractures by both readers as compared to original MDCT. Further decreasing the number of projections down to $10 \%$ of the original data allowed for correct detection of $95.8 \%$ (R1) and $91.7 \%$ (R2) of vertebral fractures (Table 4).

Both readers reported preserved high diagnostic confidence for both virtual lowering of tube current and lowered projection numbers down to $50 \%$ of original MDCT without a significant difference in scores $(p=$ 0.48 for R1 and $p=0.41$ for R2; Table 4 and Fig. 4). For MDCT with $25 \%$ or $10 \%$ of original projections, average diagnostic confidence was still high (D100 P25) to medium (D100 P10), and it was medium (D25 P100) to low (D10 P100) when MDCT with virtually lowered tube current was considered (Table 4 and Fig. 4). Correspondingly, a significant difference was observed between MDCT with virtually lowered tube current and sparse-sampled imaging at $25 \%$ or $10 \%$ of original tube current or projections $(p<0.001$ for both readers; Table 4 and Fig. 4). Excellent agreement between the evaluations of both readers was observed for both virtual tube current reductions and sparse sampling down to $10 \%$ of projections of original imaging data (ICC >0.90, R1 vs. R2 for D50 P100/D25 P100/D10 P100 and D100 P50/D100 P25/ D100 P10; Table 4).

Concerning the age of reported vertebral fractures, sparse sampling showed better results regarding the differentiation between acute, old, and unclear fracture age (Table 4). For sparse-sampled MDCT at $25 \%$ of original projections, fracture age was determined as unclear in $4.3 \%$ (R1) and $6.7 \%$ (R2) of detected vertebral fractures. According to imaging with $25 \%$ of original tube current, $42.6 \%(\mathrm{R} 1)$ and $48.9 \%$ (R2) of detected vertebral fractures were of unclear age (Table 4). Excellent agreement was observed in the evaluations of fracture age between readers (kappa >0.88; Table 4). 


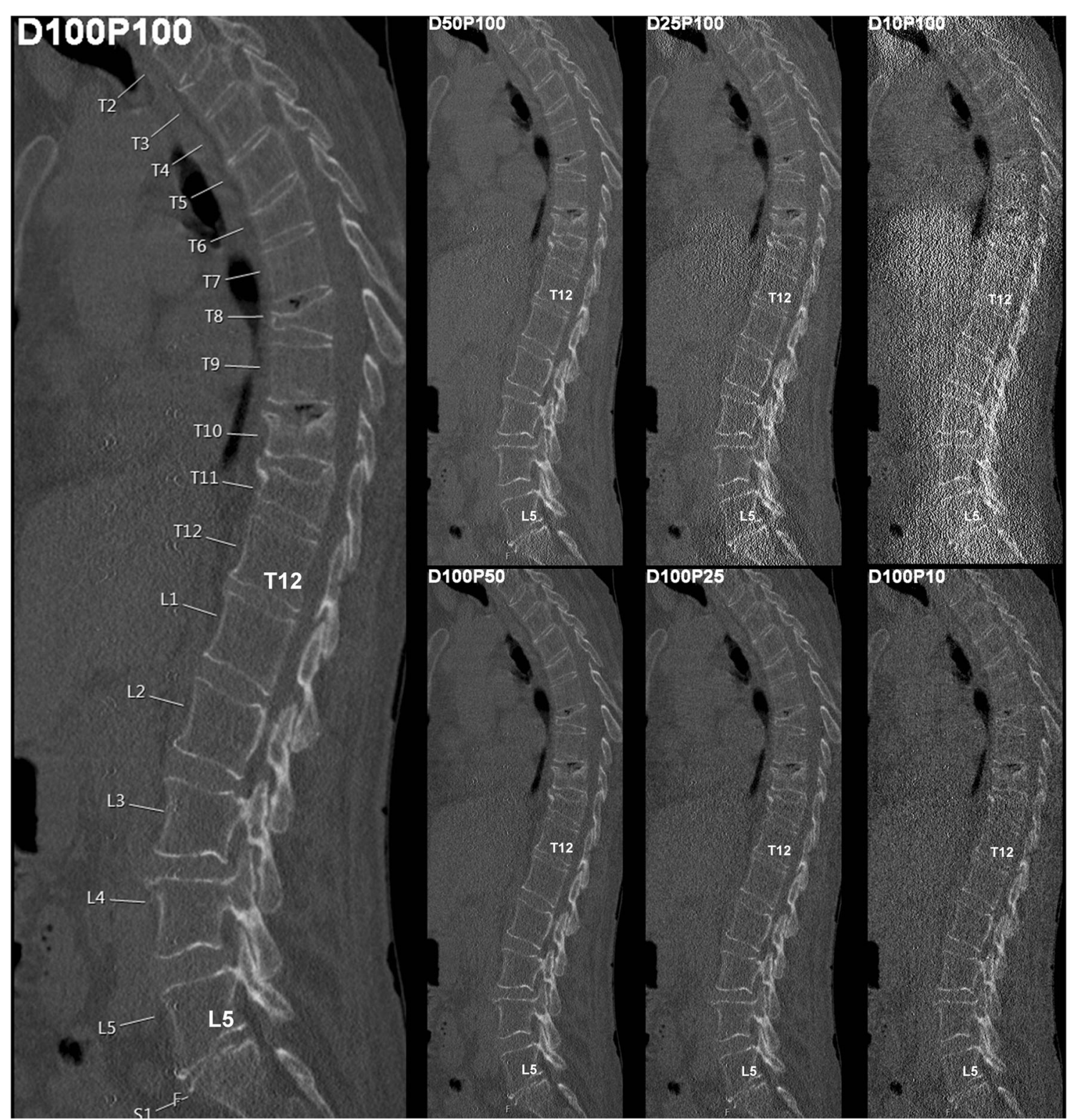

Fig. 2 Virtual tube current reduction and sparse sampling in multidetector CT (MDCT) of the thoracic and lumbar spine. Sagittal slices derived from full-dose MDCT (D100 P100), MDCT with virtually lowered tube current (D50 P100, D25 P100, and D10 P100), and

\section{Discussion}

This study investigated the effects of virtual tube current reduction and sparse sampling on image quality and diagnostic accuracy of vertebral fractures in MDCT. When comparing virtual tube current reductions to sparse sampling, superior results for image quality and fracture diagnostics were evident for sparse-sampled MDCT. Specifically, no missed vertebral fractures occurred for MDCT with a reduction of 50\% in projection numbers, and determination of fracture age was still reliably possible in MDCT with a reduction in projection numbers of $75 \%$.
MDCT with sparse sampling (D100 P50, D100 P25, and D100 P10) are shown in a patient with five thoracic fractures (T3, T6, T8, T10, and T11)

$\mathrm{CT}$ is increasingly applied for first-line diagnostics of vertebral fractures due to its high sensitivity and specificity and excellent fracture detection rates [4-6]. However, clearly higher estimated effective doses of $5.6 \mathrm{mSv}$ for the lumbar and $10.0 \mathrm{mSv}$ for the whole dorsal spine in CT compared with radiography result in a considerably increased risk of developing cancer later in life [7-9]. Importantly, cancer risks are summative, and radiography or CT performed for initial diagnostics are not the only sources of radiation exposure, with a patient suffering from an acute traumatic vertebral fracture being exposed to a cumulative effective dose of about $38 \mathrm{mSv}$ only 
Table 3 Overall image evaluation

\begin{tabular}{|c|c|c|c|c|c|c|c|c|c|c|}
\hline \multicolumn{11}{|c|}{ Overall Image Quality } \\
\hline & D100 P100 & D50 P100 & D100 P50 & $p$-value & D25 P100 & D100 P25 & $p$-value & D10 P100 & D100 P10 & $p$-value \\
\hline $\mathrm{R} 1$ & $1.03 \pm 0.17$ & $1.74 \pm 0.56$ & $1.31 \pm 0.47$ & $<0.001$ & $2.80 \pm 0.68$ & $2.17 \pm 0.45$ & $<0.001$ & $3.91 \pm 0.51$ & $3.23 \pm 0.43$ & $<0.001$ \\
\hline $\mathrm{R} 2$ & $1.03 \pm 0.17$ & $1.77 \pm 0.55$ & $1.31 \pm 0.47$ & $<0.001$ & $2.89 \pm 0.58$ & $2.20 \pm 0.47$ & $<0.001$ & $3.97 \pm 0.57$ & $3.26 \pm 0.44$ & $<0.001$ \\
\hline Interreader ICC & - & 0.98 & 0.93 & - & 0.94 & 0.97 & - & 0.89 & 0.96 & - \\
\hline \multicolumn{11}{|l|}{ Overall Artifacts } \\
\hline & D100 P100 & D50 P100 & D100 P50 & $p$-value & D25 P100 & D100 P25 & $p$-value & D10 P100 & D100 P10 & $p$-value \\
\hline $\mathrm{R} 1$ & $1.17 \pm 0.38$ & $1.86 \pm 0.69$ & $1.57 \pm 0.56$ & $<0.001$ & $2.71 \pm 0.67$ & $2.17 \pm 0.38$ & $<0.001$ & $4.03 \pm 0.71$ & $2.97 \pm 0.51$ & $<0.001$ \\
\hline $\mathrm{R} 2$ & $1.17 \pm 0.38$ & $1.86 \pm 0.65$ & $1.60 \pm 0.60$ & 0.20 & $2.86 \pm 0.65$ & $2.20 \pm 0.47$ & $<0.001$ & $4.14 \pm 0.69$ & $3.14 \pm 0.65$ & $<0.001$ \\
\hline Interreader ICC & - & 0.93 & 0.88 & - & 0.91 & 0.87 & - & 0.94 & 0.81 & - \\
\hline \multicolumn{11}{|c|}{ Contrast of Vertebrae } \\
\hline & D100 P100 & D50 P100 & D100 P50 & $p$-value & D25 P100 & D100 P25 & $p$-value & D10 P100 & D100 P10 & $p$-value \\
\hline $\mathrm{R} 1$ & $1.17 \pm 0.38$ & $1.69 \pm 0.53$ & $1.17 \pm 0.38$ & $<0.001$ & $2.63 \pm 0.69$ & $2.11 \pm 0.58$ & $<0.001$ & $3.77 \pm 0.77$ & $2.91 \pm 0.56$ & $<0.001$ \\
\hline $\mathrm{R} 2$ & $1.17 \pm 0.38$ & $1.71 \pm 0.57$ & $1.26 \pm 0.44$ & $<0.001$ & $2.66 \pm 0.77$ & $2.29 \pm 0.67$ & 0.005 & $3.89 \pm 0.68$ & $3.06 \pm 0.59$ & $<0.001$ \\
\hline Interreader ICC & - & 0.92 & 0.86 & - & 0.96 & 0.88 & - & 0.94 & 0.88 & - \\
\hline
\end{tabular}

Results of overall image evaluation as mean \pm standard deviation for overall image quality, overall artifacts, and contrast of vertebrae according to the evaluation of reader 1 (R1) and reader 2 (R2). Results are separately provided for multi-detector CT (MDCT) with virtually lowered tube current (D50 P100, D25 P100, and D10 P100) and sparse-sampled MDCT (D100 P50, D100 P25, and D100 P10) as well as original imaging (D100 P100). Interreader intraclass correlation coefficients (ICCs) and $p$ values are shown for the comparison of MDCT with virtually lowered tube current against sparse-sampled MDCT

during inpatient stay and without taking into account later follow-up imaging [32]. Consequently, reduction of CT-related radiation exposure is necessary, but should ideally be achieved without loss of image quality or diagnostic accuracy. Despite evident clinical relevance, only a limited body of literature distinctly evaluated approaches for radiation exposure reductions in $\mathrm{CT}$ of the spine.

Concerning CT with reduced doses, a small increase in image noise and no difference in subjective image quality evaluation was reported for cervical structures, allowing dose reductions of $61-71 \%$ [11]. Low-kV CT with reduced radiation doses by approximately $34 \%$ demonstrated good image quality for structures of the neck, but compromised image quality for the lower cervical spine [10]. In patients with lumbar disc herniation, simulated low-dose CT with reductions in tube charge settings to $65 \%$ of the standard dose were considered adequate for diagnostic purposes previously [33]. Furthermore, IR algorithms have been introduced for $\mathrm{CT}$ with reduced doses, leading to better image quality for intervertebral discs, neural foramina, and ligaments, but worse image quality for vertebrae when compared with standard-dose CT using FBP [12]. Ultra-low-dose CT may still provide an acceptable image quality and exhibited a diagnostic accuracy similar to that of lowdose CT in patients with chronic lumbar back pain [34].

To the authors' knowledge, only few recent studies investigated $\mathrm{CT}$ with reduced doses specifically for diagnostics of vertebral fractures. The diagnostic performance of lumbar low-dose CT (47-69\% radiation dose reduction) combined with IR was comparable to that of standard-dose CT with IR [13]. Higher levels of IR for low-dose CT (50\% radiation dose reduction) still provided high image quality and diagnostic confidence [14]. In contrast to these studies, we simulated tube current reduction, which allows for intra-subject comparisons between standard- and low-dose MDCT with virtually lowered tube currents down to even $10 \%$ of original imaging. Thus, low-dose MDCT was simulated with relative dose reduction steps in comparison to original MDCT, which enables systematic virtual tube current reductions as a fraction of the initially performed, optimal scanning protocol according to the scanner's automatic tube current modulation.

We further applied sparse sampling, which is novel for diagnostics of vertebral fractures. So far, assessments of bone mineral density and microstructure at the spine derived from MDCT with sparse sampling have been performed, with sparse-sampled imaging appearing more robust in comparison to MDCT with virtually lowered tube currents $[16,17]$. In the present study, sparse sampling was superior in terms of overall image quality, overall artifacts, and contrast of vertebrae when compared with MDCT with virtually lowered tube current (Table 3 and Fig. 3). These results were obtained with good to excellent correlations between two experienced readers for MDCT with virtually lowered tube currents 
Fig. 3 Overall image evaluation. This figure depicts the mean scores \pm standard deviation for overall image quality, overall artifacts, and contrast of vertebrae according to the evaluation of reader 1 (R1) and reader 2 (R2). Blank circles show results for multi-detector CT (MDCT) with virtually lowered tube current (D50 P100, D25 P100, and D10 P100), whereas black circles visualize the results derived from sparse-sampled MDCT (D100 P50, D100 P25, and D100 P10). The black square represents the results for original MDCT with $100 \%$ of tube current and $100 \%$ of projections (D100 P100)

and sparse sampling, respectively (Table 3 and Fig. 3). Furthermore, sparse sampling led to superior results in detecting vertebral fractures, with no missed fractures for D100 P50 in contrast to D50 P100 (Table 4). Diagnostic confidence and correct determination of fracture age was better for sparse sampling, with clinically acceptable determination of fracture age in D100 P25 compared with D25 P100 (Table 4 and Fig. 4).

There are limitations to this study. First, it is not yet possible to apply sparse sampling at commercial MDCT scanners, thus restricting direct clinical applicability. However, first results from a prototype were recently reported, indicating that sparse sampling for MDCT could become broadly available in future generations of MDCT scanners [19, 20]. Second, we used FBP instead of IR, but IR has the potential to provide increased image quality particularly for imaging with reduced doses [35-37]. The use of algorithms taking advantage of artificial intelligence for image reconstructions might further regularly improve image quality in the near future [37]. Third, we solely enrolled patients with vertebral fractures and without implants, such as spinal instrumentation. Thus, upcoming studies may evaluate sparse sampling in cohorts with spinal implants to distinctly evaluate whether sparse sampling is also beneficial and even superior to tube current restrictions when implant-related metal artifacts are present in MDCT. Fourth, the retrospective design and the comparatively small patient cohort have to be acknowledged as a limitation. Prospective approaches including more patients are needed to confirm the results of the present study.

In conclusion, our results demonstrate the feasibility of using sparse sampling for fracture detection at the spine, with clear superiority as compared to MDCT with virtual reduction of tube current. Therefore, sparse sampling represents a promising option that might allow for even more drastic radiation dose reductions while revealing better image quality and diagnostic characteristics than MDCT with tube current reduction does.
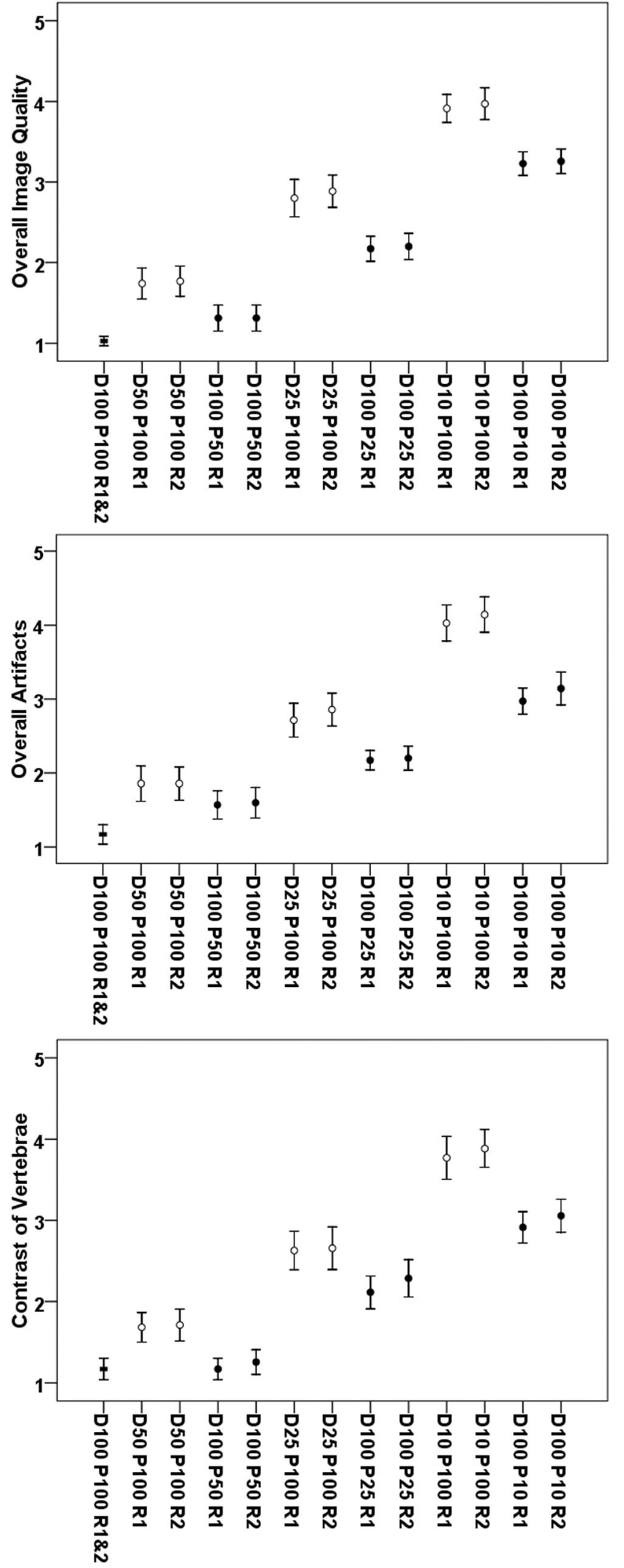
Table 4 Fracture evaluation

\begin{tabular}{|c|c|c|c|c|c|c|c|c|c|c|}
\hline \multicolumn{11}{|l|}{ Reported Number of Fractures } \\
\hline & D100 P100 & D50 P100 & D100 P50 & - & D25 P100 & D100 P25 & - & D10 P100 & D100 P10 & - \\
\hline R1 & 48 & 48 & 48 & & 47 & 47 & & 38 & 46 & \\
\hline $\mathrm{R} 2$ & 48 & 46 & 48 & & 45 & 45 & & 42 & 44 & \\
\hline \multicolumn{11}{|l|}{ Diagnostic Confidence } \\
\hline & D100 P100 & D50 P100 & D100 P50 & $p$-value & D25 P100 & D100 P25 & $p$-value & D10 P100 & D100 P10 & $p$-value \\
\hline $\mathrm{R} 1$ & $1.02 \pm 0.14$ & $1.17 \pm 0.38$ & $1.13 \pm 0.39$ & 0.48 & $1.83 \pm 0.79$ & $1.34 \pm 0.48$ & $<0.001$ & $2.55 \pm 0.69$ & $1.87 \pm 0.58$ & $<0.001$ \\
\hline $\mathrm{R} 2$ & $1.02 \pm 0.14$ & $1.15 \pm 0.36$ & $1.10 \pm 0.31$ & 0.41 & $1.80 \pm 0.79$ & $1.29 \pm 0.46$ & $<0.001$ & $2.45 \pm 0.71$ & $1.80 \pm 0.51$ & $<0.001$ \\
\hline Interreader ICC & - & 0.91 & 0.96 & - & 0.98 & 0.91 & - & 0.97 & 0.98 & - \\
\hline \multicolumn{11}{|c|}{ Age of Fracture (acute / unclear / old) } \\
\hline & D100 P100 & D50 P100 & D100 P50 & Kappa & D25 P100 & D100 P25 & Kappa & D10 P100 & D100 P10 & Kappa \\
\hline $\mathrm{R} 1$ & $28 / 0 / 20$ & $27 / 2 / 19$ & $27 / 1 / 20$ & 0.84 & $18 / 20 / 9$ & $26 / 2 / 19$ & 0.42 & $4 / 30 / 4$ & $16 / 24 / 6$ & 0.24 \\
\hline $\mathrm{R} 2$ & $28 / 0 / 20$ & $27 / 3 / 16$ & $26 / 1 / 21$ & 0.79 & $16 / 22 / 7$ & $25 / 3 / 17$ & 0.35 & $4 / 34 / 4$ & $15 / 24 / 5$ & 0.13 \\
\hline Interreader Weighted Kappa & - & 0.96 & 0.92 & - & 0.93 & 0.96 & - & 0.89 & 0.92 & - \\
\hline
\end{tabular}

Results regarding the reported number of fractures, diagnostic confidence (mean \pm standard deviation), and age of fracture (absolute numbers for acute/ unclear/old vertebral fractures) according to the evaluation of multi-detector CT (MDCT) by reader 1 (R1) and reader 2 (R2). Results are separately provided for virtually lowered tube current (D50 P100, D25 P100, and D10 P100), sparse-sampled MDCT (D100 P50, D100 P25, and D100 P10), and the original dose (D100 P100). Interreader intraclass correlation coefficients (ICCs) and $p$ values derived from the comparison of MDCT with virtually lowered tube current against sparse-sampled MDCT are provided for diagnostic confidence. Cohen's kappa coefficients are depicted for agreement of reported age of detected vertebral fractures between MDCT with virtually lowered tube current and sparse-sampled MDCT, and weighted Cohen's kappa is shown for interreader evaluation

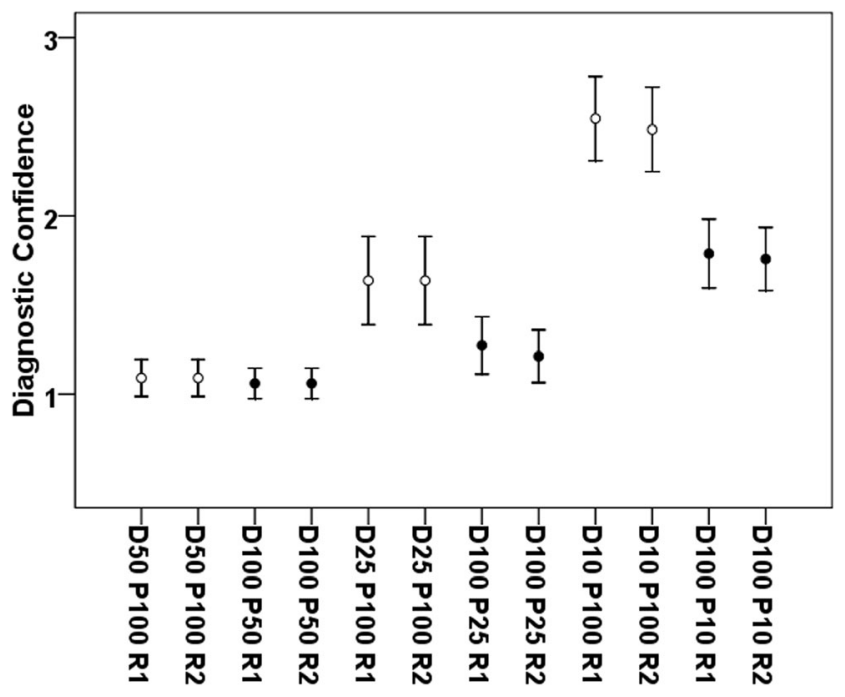

Fig. 4 Diagnostic confidence. This figure depicts the mean scores \pm standard deviation for diagnostic confidence according to the evaluation of reader 1 (R1) and reader 2 (R2). Blank circles show results for multidetector CT (MDCT) with virtually lowered tube current (D50 P100, D25 $\mathrm{P} 100$, and D10 P100), whereas black circles visualize the results derived from sparse-sampled MDCT (D100 P50, D100 P25, and D100 P10)
Funding This study has received support from Philips Healthcare and funding from the European Research Council (ERC) under the European Union's Horizon 2020 research and innovation programme (grant agreement No 637164-iBack-ERC-2014-STG) and the Nivida Corporation. We further acknowledge support through the German Department of Education and Research (BMBF) under grant IMEDO (13GW0072C) and the German Research Foundation (DFG) within the Research Training Group GRK 2274.

\section{Compliance with ethical standards}

Guarantor The scientific guarantor of this publication is Thomas Baum, MD.

Conflict of interest The authors of this manuscript declare no relationships with any companies, whose products or services may be related to the subject matter of the article.

Statistics and biometry One of the authors has significant statistical expertise.

Informed consent Written informed consent was not required for this study because of its retrospective character and the analyses being based only on data acquired for clinical routine.

Ethical approval Institutional Review Board approval was obtained.

\section{Methodology}

- Retrospective

- Diagnostic or prognostic study

- Performed at one institution 
Open Access This article is distributed under the terms of the Creative Commons Attribution 4.0 International License (http:// creativecommons.org/licenses/by/4.0/), which permits unrestricted use, distribution, and reproduction in any medium, provided you give appropriate credit to the original author(s) and the source, provide a link to the Creative Commons license, and indicate if changes were made.

\section{References}

1. Cooper C, Atkinson EJ, O'Fallon WM, Melton LJ 3rd (1992) Incidence of clinically diagnosed vertebral fractures: a populationbased study in Rochester, Minnesota, 1985-1989. J Bone Miner Res 7:221-227

2. Schousboe JT (2016) Epidemiology of vertebral fractures. J Clin Densitom 19:8-22

3. Van der Klift M, De Laet CE, McCloskey EV, Hofman A, Pols HA (2002) The incidence of vertebral fractures in men and women: the Rotterdam Study. J Bone Miner Res 17:1051-1056

4. Venkatesan M, Fong A, Sell PJ (2012) CT scanning reduces the risk of missing a fracture of the thoracolumbar spine. J Bone Joint Surg Br 94:1097-1100

5. Harris TJ, Blackmore CC, Mirza SK, Jurkovich GJ (2008) Clearing the cervical spine in obtunded patients. Spine (Phila Pa 1976) 33: $1547-1553$

6. Shah LM, Ross JS (2016) Imaging of spine trauma. Neurosurgery 79:626-642

7. Richards PJ, George J, Metelko M, Brown M (2010) Spine computed tomography doses and cancer induction. Spine (Phila Pa 1976) 35:430-433

8. Richards PJ, George J (2010) Diagnostic CT radiation and cancer induction. Skeletal Radiol 39:421-424

9. Brenner DJ, Hall EJ (2007) Computed tomography-an increasing source of radiation exposure. N Engl J Med 357:2277-2284

10. Gnannt R, Winklehner A, Goetti R, Schmidt B, Kollias S, Alkadhi $\mathrm{H}$ (2012) Low kilovoltage CT of the neck with $70 \mathrm{kVp}$ : comparison with a standard protocol. AJNR Am J Neuroradiol 33:1014-1019

11. Mulkens TH, Marchal P, Daineffe S et al (2007) Comparison of low-dose with standard-dose multidetector CT in cervical spine trauma. AJNR Am J Neuroradiol 28:1444-1450

12. Becce F, Ben Salah Y, Verdun FR et al (2013) Computed tomography of the cervical spine: comparison of image quality between a standard-dose and a low-dose protocol using filtered backprojection and iterative reconstruction. Skeletal Radiol 42:937-945

13. Lee SH, Yun SJ, Kim DH, Jo HH, Song JG, Park YS (2017) Diagnostic usefulness of low-dose lumbar multi-detector CT with iterative reconstruction in trauma patients: a comparison with standard-dose CT. Br J Radiol 90:20170181

14. Weinrich JM, Well L, Regier M et al (2018) MDCT in suspected lumbar spine fracture: comparison of standard and reduced dose settings using iterative reconstruction. Clin Radiol 73:675 e679675 e 615

15. Abbas S, Lee T, Shin S, Lee R, Cho S (2013) Effects of sparse sampling schemes on image quality in low-dose CT. Med Phys 40:111915

16. Mei K, Kopp FK, Bippus R et al (2017) Is multidetector CT-based bone mineral density and quantitative bone microstructure assessment at the spine still feasible using ultra-low tube current and sparse sampling? Eur Radiol 27:5261-5271
17. Mookiah MRK, Subburaj K, Mei K et al (2018) Multidetector computed tomography imaging: effect of sparse sampling and iterative reconstruction on trabecular bone microstructure. J Comput Assist Tomogr 42:441-447

18. Koesters T, Knoll F, Sodickson A, Sodickson DK, Otazo R (2017) SparseCT: interrupted-beam acquisition and sparse reconstruction for radiation dose reduction. Proc. SPIE 10132, Medical Imaging 2017: Physics of Medical Imaging, 101320Q. https://doi.org/10. $1117 / 12.2255522$

19. Chen B, Muckley M, Sodickson A et al (2018) First multislit collimator prototype for sparseCT: design, manufacturing and initial validation The Fifth International Conference on Image Formation in X-Ray Computed Tomography, Salt Lake City, pp 52-55

20. Muckley M, Chen B, O'Donnell T et al (2018) Reconstruction of reduced-dose sparseCT data acquired with an interruped-beam prototype On A clinical scanner The Fifth International Conference on Image Formation in X-Ray Computed Tomography, Salt Lake City, pp 56-59

21. Zabic S, Wang Q, Morton T, Brown KM (2013) A low dose simulation tool for CT systems with energy integrating detectors. Med Phys 40:031102

22. Muenzel D, Koehler T, Brown K et al (2014) Validation of a low dose simulation technique for computed tomography images. PLoS One 9:e107843

23. Sollmann N, Mei K, Schwaiger BJ et al (2018) Effects of virtual tube current reduction and sparse sampling on MDCT-based femoral BMD measurements. Osteoporos Int. https://doi.org/10.1007/ s00198-018-4675-6

24. Zhao Z, Gang GJ, Siewerdsen JH (2014) Noise, sampling, and the number of projections in cone-beam CT with a flat-panel detector. Med Phys 41:061909

25. Ramachandran GN, Lakshminarayanan AV (1971) Threedimensional reconstruction from radiographs and electron micrographs: application of convolutions instead of Fourier transforms. Proc Natl Acad Sci U S A 68:2236-2240

26. Fehringer A, Lasser T, Zanette I, Noel PB, Pfeiffer F (2014) A versatile tomographic forward- and back-projection approach on multi-GPUs. Proc. SPIE 9034, Medical Imaging 2014: Image Processing, 90344F. https://doi.org/10.1117/12.2043860

27. Shrout PE, Fleiss JL (1979) Intraclass correlations: uses in assessing rater reliability. Psychol Bull 86:420-428

28. Bartko JJ (1966) The intraclass correlation coefficient as a measure of reliability. Psychol Rep 19:3-11

29. Cohen J (1968) Weighted kappa: nominal scale agreement with provision for scaled disagreement or partial credit. Psychol Bull 70:213-220

30. Cohen J (1960) A coefficient of agreement for nominal scales. Educ Psychol Meas 20:37-46

31. Sim J, Wright CC (2005) The kappa statistic in reliability studies: use, interpretation, and sample size requirements. Phys Ther 85: 257-268

32. Martin E, Prasarn M, Coyne E et al (2013) Inpatient radiation exposure in patients with spinal trauma. J Spinal Cord Med 36:112117

33. Bohy P, de Maertelaer V, Roquigny A, Keyzer C, Tack D, Gevenois PA (2007) Multidetector CT in patients suspected of having lumbar disk herniation: comparison of standard-dose and simulated lowdose techniques. Radiology 244:524-531

34. Lee SH, Yun SJ, Jo HH, Kim DH, Song JG, Park YS (2018) Diagnostic accuracy of low-dose versus ultra-low-dose CT for lumbar disc disease and facet joint osteoarthritis in patients with low back pain with MRI correlation. Skeletal Radiol 47:491-504 
35. Beister M, Kolditz D, Kalender WA (2012) Iterative reconstruction methods in X-ray CT. Phys Med 28:94-108

36. Willemink MJ, Leiner T, de Jong PA et al (2013) Iterative reconstruction techniques for computed tomography part 2: initial results in dose reduction and image quality. Eur Radiol 23: $1632-1642$
37. Willemink MJ, Noel PB (2018) The evolution of image reconstruction for CT-from filtered back projection to artificial intelligence. Eur Radiol. https://doi.org/10.1007/s00330-018-5810-7

Publisher's note Springer Nature remains neutral with regard to jurisdictional claims in published maps and institutional affiliations. 